\title{
Effect of ball milling on the production of nanocellulose using mild acid
}

\section{hydrolysis method}

\author{
Patchiya Phanthong ${ }^{1}$, Guoqing Guan ${ }^{1,2^{*}}$, Yufei Ma ${ }^{1}$, Xiaogang $\mathrm{Hao}^{3}$, Abuliti Abudula ${ }^{1,2}$ \\ ${ }^{1}$ Graduate School of Science and Technology, Hirosaki University, 1-Bunkyocho, Hirosaki \\ 036-8560, Japan \\ ${ }^{2}$ North Japan Research Institute for Sustainable Energy (NJRISE), Hirosaki University, 2-1-3, \\ Matsubara, Aomori 030-0813, Japan \\ ${ }^{3}$ Department of Chemical Engineering, Taiyuan University of Technology, Taiyuan 030024,
}

\section{China}

${ }^{*}$ Corresponding author. Tel.: +81-17-762-7756; Fax: +81-17-735-5411.

E-mail address: guan@hirosaki-u.ac.jp (G. Guan) 


\section{ABSTRACT}

Nanocellulose was extracted from two kinds of general cellulose feedstocks, i.e., cellulose paper and cellulose powder, by coupling planetary dry ball milling with mild acid hydrolysis.

The effect of ball milling time on the yield and properties of nanocellulose obtained by mild hydrolysis in lower concentration $(47 w t \%)$ of sulfuric acid was investigated in details. The obtained nanocellulose was characterized by scanning electron microscopy (SEM), Fourier transform infrared spectroscopy $(F T I R)$, X-ray diffraction $(X R D)$, and thermogravimetric analysis $(T G A)$. It is found that the crystallinity and crystal size of ball-milled cellulose decreased with the increase of ball milling time, and the mild acid hydrolysis of the ball-milled cellulose resulted in the crystallinity and thermal stability of nanocellulose at the high temperature range increased but without any changes of chemical structure. It indicates that the appropriate ball-milling of cellulose feedstock could be benefit for the mild hydrolysis process for the production of high quality nanocellulose with high yield.

Keywords Nanocellulose; Cellulose; Sulfuric acid; Ball milling; Mild acid hydrolysis. 


\section{1.\# Introduction}

In our daily life, fabrics, ropes, paper and many other consumer goods are made of natural fibers. Most of natural fibers from plants basically consists of cellulose [1], which is the main structure of plant cell wall. Cellulose fiber generally contains two types of structures, i.e., highly ordered (crystalline) and disordered (amorphous) ones [2, 3]. Extraction of the crystalline region from microfibrillated cellulose generally results in nanocellulose.

Nanocellulose is considered as a new class of eco-material with many advantages, such as nanoscale dimension, renewability, high surface area, specific high strength and modulus, unique morphology and good optical properties. Thus, it can be used in many fields such as nanocomposite materials, drug delivery, food additive, and filling materials in rubber industry [2, 4]. That is why nanocellulose extraction from biomass is an interesting study field nowadays. To date, acid hydrolysis is one of preferential processes to remove the amorphous regions within the cellulose microfibrils, in which sulfuric acid is the most generally used acid because it produces negative charges on the particles, leading to more stable suspension $[3,5]$.

Many previous studies indicate that the typical concentration of sulfuric acid in hydrolysis reaction for extraction of nanocellulose is near $65 w t \%$ [4]. Dong et al. extracted cellulose microcrystallite from Whatman No.1 filter paper powder (cotton cellulose) using $64 w t \%$ of sulfuric acid with a pulp to acid ratio of $1: 8.75$ at $45{ }^{\circ} \mathrm{C}$ for $1 h$ [6]. Haafiz et al. isolated 
cellulose nanowhiskers from microcrystalline cellulose $(M C C)$ of oil palm biomass by using 64 $w t \%$ sulfuric acid hydrolysis with an acid to $\mathrm{MCC}$ ratio of $8.75 \mathrm{~mL} / \mathrm{g}$ at $40{ }^{\circ} \mathrm{C}$ for $1 \mathrm{~h}$ [7]. Using lower concentration of sulfuric acid hydrolysis will be more attractive for nanocellulose extraction due to more environmentally friendly and less wastewater from washing step, which will decrease the production cost.

Mechanical process is one of approaches to diminish cellulosic fibers into nanocelluloses, by which the cellulose size can be reduced by splitting along the longitudinal axis of the cellulose structures via the mechanical stress $[3,8]$. Planetary ball mill is such mechanical techniques, in which the artificial gravity generated by the centrifugal force can be applied to the grinding medium. It is found that the crystallinity of cellulose decreases with the dry ball mill, and as such the nanocellulose could be more easily extracted from the ball-milled cellulose[9-13].

In this study, two general kinds of cellulose, i.e., cellulose paper and cellulose powder, were selected as the raw materials for nanocellulose extraction. To improve the extraction efficiency, the raw materials were pretreated by planetary ball mill at first and then, mild hydrolysis of the ball-milled cellulose with lower concentration of sulfuric acid at lower reaction temperature for a shorter reaction time was performed. The obtained nanocellulose was characterized by using SEM, FTIR, XRD and TGA for investigation of its morphology, the change of chemical composition, crystalline allomorph, crystallinity and crystal size, and thermal stability, 
respectively. It is expected that such a kind of method can effectively extract nanocellulose from cellulose feedstock.

\section{2.\# Material and methods}

\subsection{Materials}

Grade 3MM Chr cellulose chromatography paper (Whatman), $38 \mu \mathrm{m}$ (400 mesh) size of cellulose powder (Wako), and sulfuric acid aqueous solution (47 wt $\%$ ) were purchased from Wako Pure Chemical Industries Ltd., Japan. The cellulose paper $\left(C P P^{1}\right)$ was crushed by blender for 30 seconds to small pieces prior to use while the cellulose powder $\left(C P D^{2}\right)$ was used as received.

\subsection{Ball milling pretreatment}

Cellulose materials were ball-milled with an ITO LP-1 Planetary pot mill. $80 \mathrm{~mL}$ of jar with the three different diameters of zirconia ball $(10,5$, and $2 \mathrm{~mm})$ was used, in which the ball to material weight ratio $(B M R)$ was $12: 1$ and the weight ratio of the balls with 10,5 , and $2 \mathrm{~mm}$ diameters was 5:4:3. In the preliminary experiments, it is found that high rotation speed resulted in very low nanocellulose yield. Thus, in this study, a relative low rotation speed of $300 \mathrm{rpm}$ was

1 CPP: Cellulose Paper

2 CPD: Cellulose Powder 
selected. The ball milling was carried out for $0.5,1,2$, and $3 h$ at room temperature in order to investigate the effect of ball milling time. The pretreated cellulose materials are defined as Ball-milled Cellulose Paper $\left(B M C P P_{\mathrm{x}}{ }^{3}\right)$ or Ball-milled Cellulose Powder $\left(B M C P D_{\mathrm{x}}{ }^{4}\right)$ corresponds to the type of material, while $\mathrm{x}$ is the ball milling pretreatment time $(x=0.5,1,2$, or $3 h)$

\subsection{Acid hydrolysis}

Acid hydrolysis was performed by using a low concentration of sulfuric acid aqueous solution (47 wt\%). $8.8 \mathrm{~mL}$ of acid/1 $\mathrm{g}$ of cellulose materials was used in each case. The reaction temperature was controlled at $45{ }^{\circ} \mathrm{C}$ with a water bath and the mixing speed was set at $600 \mathrm{rpm}$ The reaction time is fixed at 90 minutes.

The sulfuric acid hydrolysis was stopped by adding 10 -fold cold distilled water $\left(4{ }^{\circ} \mathrm{C}\right)$. The suspension was centrifuged at $8500 \mathrm{rpm}$ for 10 minutes to get the precipitates and simultaneously remove out the excess acid. The precipitate was then suspended in distilled water, followed by centrifugation. This process was repeated until the neutral $\mathrm{pH}$ was achieved. Subsequently, the suspension was frozen in freezer at $-30{ }^{\circ} \mathrm{C}$, then dried in freeze-dryer. The dried product was stored in vacuum for further characterizations. The obtained products are

${ }^{3} B M C P P_{x}$ : Ball-milled cellulose paper at $\mathrm{x} h$ of ball milling time

${ }^{4} B M C P D_{x}$ : Ball-milled cellulose powder at $\mathrm{x} h$ of ball milling time 
defined as Nanocellulose from cellulose materials; Nanocellulose from cellulose paper $\left(N C C P P^{5}\right)$, Nanocellulose from cellulose powder $\left(N C C P D^{6}\right)$, Nanocellulose from ball-milled cellulose paper at $\mathrm{x} h$ of ball milling pretreatment time $\left(N C B M C P P_{x}^{7}\right)$, and Nanocellulose from ball-milled cellulose powder at $\mathrm{x} h$ of ball milling pretreatment time $\left(N C B M C P D_{x}{ }^{8}\right)$.

\subsection{Characterization}

Surface morphologies of samples were examined by a scanning electron microscope (SEM, SU8010, Hitachi) at an acceleration voltage of $1.0 \mathrm{kV}$. A drop of diluted product suspension was deposited on the carbon tape and air-dried at $50{ }^{\circ} \mathrm{C}$ oven for $2 h$. Then, the sample was sputter-coated with Pt at $15 m A$ for 20 seconds to avoid charging.

Fourier transform infrared spectroscopy $(F T-I R)$ was recorded by using Jasco FT/IR-4200 infrared spectrophotometer with wavelengths in the range of $500-4000 \mathrm{~cm}^{-1}$, in which the sample was ground into fine powder and mixed with $\mathrm{KBr}$ followed by pressing the mixture into thin pellet.

To study the crystallinity, X-ray diffraction $(X R D)$ was carried out on a Rigaku Smartlab X-ray diffractometer with $C u K \alpha$ radiation at $45 \mathrm{kV}$ and $200 \mathrm{~mA}$ from $10-50^{\circ}$ (28 angle range).

5 NCCPP: Nanocellulose from cellulose paper without ball milling pretreatment

${ }_{7}^{6} N C C P D$ : Nanocellulose from cellulose powder without ball milling pretreatment

${ }^{7} N C B M C P P_{x}$ : Nanocellulose from ball-milled cellulose paper at $\mathrm{x} h$ of ball milling time

${ }^{8} N C B M C P D_{x}$ : Nanocellulose from ball-milled cellulose powder at $\mathrm{x} h$ of ball milling time 
The crystallinity was calculated using peak height method. It is calculated from the height ratio between the intensity of the crystalline peak and the total intensity after the subtraction of the background signal (non-crystalline) according to the following equation:

$$
C(\%)=100 \times \frac{I_{200}-I_{n o n-c r}}{I_{200}}
$$

where $C$ is the apparent crystallinity [\%], $I_{200}$ is the maximum intensity of the peak corresponding to the plane in the sample with the Miller indices 200 at a $2 \theta$ angle between 22-24 degrees and $I_{n o n-c r}$ represents the intensity of diffraction of the non-crystalline material, which is taken at an angle of about $18^{\circ}(2 \theta)$ in the valley between the peaks [14].

The Scherrer equation was used to calculate the crystallite size, $t(\mathrm{~nm})$, which is determined perpendicular to the (200) planes for both cellulose I and cellulose II samples:

$$
t=\frac{0.9 \lambda}{\beta \cos \theta}
$$

where $\lambda$ is the radiation wavelength for $\mathrm{Cu}, \theta$ is the diffraction angle, and $\beta$ is the corrected angular width at half maximum intensity in radians [15].

The thermal stability of sample was determined by using a themogravimetric analyzer (DTG-60H, Shimadzu). An average mass of $10 \mathrm{mg}$ of the sample was put in an alumina cup and heated from room temperature to $600{ }^{\circ} \mathrm{C}$ at a heating rate of $10{ }^{\circ} \mathrm{C} / \mathrm{min}$ under a nitrogen flow of 
$50 \mathrm{~cm}^{3} / \mathrm{min}$. Differential thermal gravimetry $(D T G)$ was calculated based on $T G A$ values using a forward finite difference method as the following equation:

$$
\mathrm{DTG}=\frac{\left(\mathrm{w}_{\mathrm{t}+\Delta \mathrm{t}}-\mathrm{w}_{\mathrm{t}}\right)}{\Delta \mathrm{t}}
$$

where $w_{t+\Delta t}$ and $w_{t}$ are the residual weight of sample at time $t+\Delta t$ and $t$, respectively, and $\Delta t$ is the time interval for reading residual sample weight [16].

\section{3.\# Results and discussion}

\subsection{Product yield}

The weight of final product was recorded and calculated as product yield based on $100 \%$ of raw cellulose or ball-milled cellulose materials for acid hydrolysis. The yields of all final products were tabulated and shown in Table 1. The average yield of nanocellulose from $C P P$ and $B M C P P_{x}$ was $85.1 \%$, while the average yield of nanocellulose from $C P D$ and $B M C P D_{x}$ was 83.7\%. It is found that these obtained nanocelluloses yields are higher than those reported in other previous work with similar condition. Bondeson et al. isolated nanocellulose from microcrystalline cellulose by hydrolysis in $63.5 \mathrm{wt} \%$ sulfuric acid at $45^{\circ} \mathrm{C}$ for $2 \mathrm{~h}$. The yield of their obtained product was only $30 \%$ [17]. As such, mild acid hydrolysis of ball-milled cellulose can extract nanocellulose with a relative high yield. It is possible that hydrolysis of cellulose in a 
higher concentration acid could result in some nanocellulose further converted to soluble molecules.

\subsection{Morphological investigation}

The surface morphologies of $C P P, B M C P P_{x}, N C C P P$, and $N C B M C P P_{x}$ are shown in Fig. 1. Raw cellulose paper (CPP, Fig. 1(A)) showed the significant of single microfibril shape in micro scale. After ball milling pretreatment (Fig. 1(B-E)), the microfibril of raw cellulose paper was cracked in longitudinal axis and became to microfibril shape with smaller diameter and the size decreased with the increasing of ball milling time. As stated above, ball milling can produce high shear force from the collision between different sizes of balls as well as the friction of balls to wall [10] which leads to the extraction of long cellulose fibrils in longitudinal axis $[3,18]$. From the morphologies of nanocellulose products $\left(N C B M C P P_{x}\right)$ after mild acid hydrolysis (as shown in Fig. 1(F-I)), the single fibril obviously became to nanoscale size, suggesting that nanocellulose was successfully extracted from cellulose paper by using the present method. However, the morphology of $\mathrm{NCBMCPP}_{3}$ in Fig. 1(J) was found to be different from other $N C B M C P P_{x}$. It is possible due to its less crystallinity as indicated in the following sections.

The surface morphologies of $C P D, B M C P D_{x}, N C C P D$, and $N C B M C P D_{x}$ are shown in Fig. S-1 in the supplementary material. Cellulose powder $(C P D$, Fig. $\mathbf{S - 1 ( A ) ) ~ s h o w s ~ i r r e g u l a r ~ s h a p e ~}$ 
in micrometer scale. After ball milling pretreatment (Fig. S-1(B-E)), the length of cellulose powder $\left(B M C P D_{x}\right)$ became shorter. Furthermore, after the mild acid hydrolysis, nano-sized products $\left(N C B M C P D_{x}\right.$, Fig. $\left.\mathbf{S}-\mathbf{1}(\mathbf{F}-\mathbf{J})\right)$ were obtained. It also indicated that nanocellulose can be effectively extracted from cellulose powder using the present method.

\subsection{FT-IR Spectroscopic Analysis}

To investigate the change of chemical compositions occurred during the extraction process, $F T I R$ spectroscopy was measured. Fig. 2 shows $F T-I R$ spectra of (A) $C P P,(\mathrm{~B}) B M C P P_{3},(\mathrm{C})$ $N C C P P$, (D) $N C B M C P P_{0.5},(\mathrm{E}) N C B M C P P_{1},(\mathrm{~F}) N C B M C P P_{2}$, and (G) $N C B M C P P_{3}$. It should be noted that all spectra showed the same absorption peaks at around 3600-3100, 2900, 1430, 1369-1330, $900 \mathrm{~cm}^{-1}$, which are associated with the native cellulose type I. The broad peak at around 3600-3100 $\mathrm{cm}^{-1}$ attributed to the free $\mathrm{O}-\mathrm{H}$ stretching vibration of the $\mathrm{OH}$ groups while the absorption peak at around $2900 \mathrm{~cm}^{-1}$ indicated the $\mathrm{CH}_{2}$ groups of cellulose molecules. The peak appearing at $1430 \mathrm{~cm}^{-1}$ related to $\mathrm{CH}_{2}$ bending of the intermolecular hydrogen attraction at the $\mathrm{C}_{6}$ group. The absorption peaks in the region of $1369-1330 \mathrm{~cm}^{-1}$ corresponded to the $\mathrm{C}-\mathrm{H}$ and $\mathrm{C}-\mathrm{O}$ groups of polysaccharides. The detected peak at $900 \mathrm{~cm}^{-1}$ associated with the $\mathrm{C}-\mathrm{H}$ rocking vibration of $\beta$-glycosidic linkages between glucose units in cellulose [7, 19]. From all FTIR spectra, one can see that there is no significant differences in chemical compositions among cellulose paper (Fig. 2(A)), ball-milled cellulose paper (Fig. 2(B)), and nanocellulose products 
(Fig. 2(C-G)). It indicated that the mechanical ball milling pretreatment had no effect on the change of chemical composition. Moreover, the chemical compositions of nanocellulose products were also not changed after the mild acid hydrolysis reaction.

FTIR spectra related to cellulose powder are shown in Fig S-2 which describes the chemical compositions of (A) $C P D$, (B) $B M C P D_{3}$, (C) $N C C P D$, (D) $N C B M C P D_{0.5}$, (E) $N C B M C P D_{1}$, (F) $N C B M C P D_{2}$, and (G) $N C B M C P D_{3}$. All spectra also showed the similar chemical composition peaks of the native cellulose type I, which are the same as cellulose paper (Fig. 2). Moreover, there are also no significantly new compositions found in ball-milled cellulose powder and nanocellulose products. These results are agreement with the results obtained by other researchers. For example, Li et al. studied sulfuric acid hydrolysis of mulberry branch-bark cellulose, and found that the chemical composition of cellulose whiskers had no difference when compared with that of raw cellulose fibers [20].

\section{$3.4 X$-ray diffraction}

The crystalline allomorphs, crystallinity, and crystal size of samples can be examined by X-ray diffraction. Fig. 3 shows $X R D$ patterns of (A) $C P P$, (B) $B M C P P_{0.5}$, (C) $B M C P P_{1}$, (D) $B M C P P_{2}$, and (E) $\mathrm{BMCPP}_{3}$. From $X R D$ patterns of cellulose paper and its various ball-milled ones, the characteristic peaks were revealed at $2 \theta=15^{\circ}, 16.7^{\circ}, 22.9^{\circ}$, and $34.7^{\circ}$ which 
corresponded to the crystalline planes of $-110,110,200$ and 004 of the cellulose type I [21, 22]. The crystallinity and crystal size were calculated by Eqs. 1 and 2, respectively, and the results were tabulated in Table 2. Comparing with raw materials, the crystallinity and crystal size of ball-milled cellulose paper decreased with the increasing of ball milling time. The materials were cracked to smaller size after ball-milling, as supported by SEM results, which decreased the crystalline region, but increased the amorphous region [12]. For nanocellulose from cellulose paper obtained from the mild acid hydrolysis, the $X R D$ patterns are shown in Fig. 4. All five nanocellulose products, i.e., (A) $N C C P P$, (B) $N C B M C P P_{0.5}$, (C) $N C B M C P P_{1}$, (D) $N C B M C P P_{2}$, and (E) $\mathrm{NCBMCPP}_{3}$, also showed the similar characteristics of cellulose type I. This implied that the crystalline allomorphs was not changed after the mild acid hydrolysis. From the calculated crystallinity and crystal size shown in Table 2 , it is found that the crystallinity and crystal size of products increased to some extent when compared with the raw materials. This can be ascribed to the reduction and removal of amorphous cellulosic part during the acid hydrolysis reaction [23]. Thus, the remaining part in the nanocellulose should be mainly consisted of crystalline region which led to the increasing of crystallinity when compared with the ball-milled cellulose paper.

For the nanocellulose obtained from cellulose powder, Fig. $\mathbf{S}-3$ shows $X R D$ patterns of (A) $C P D$, (B) $B M C P D_{0.5}$, (C) $B M C P D_{1}$, (D) $B M C P D_{2}$, and (E) $B M C P D_{3}$. One can see that all peaks showed the characteristic peaks at $2 \theta=16^{\circ}, 22.8^{\circ}$, and $34.7^{\circ}$ which also indicated the crystal 
structure of cellulose type I [7]. In contrast, the characteristic peak of cellulose type I at $2 \theta=$ $22.8^{\circ}$ significantly decreased with the increase in the ball-milling time. This significant decrease is also shown by the calculated crystallinity and crystal size in Table 2 . It can be found that, for the products related to the ball milling time longer than $2 \mathrm{~h}$, the crystallinity decreased more than $50 \%$ when compared with that of cellulose powder. This should be also resulted from the ball-milling since the mechanical energy from the friction of balls could transfer to the cellulose and affect its crystalline structure $[10,11]$. Moreover, this result is in agreement with the study of Avolio et al. in which they used the dry ball milling to treat Arbocel $B W W 40$ cellulose fibers for $2,4,8,15,30$, and $60 \mathrm{~min}$, and found that the cellulose I crystallinity index and cellulose crystallinity decreased with the increase in ball milling time [11]. For nanocellulose products from cellulose powder, the $X R D$ patterns are shown in Fig. $\mathbf{S}-\mathbf{4}$, which describes the characteristic peaks of (A) $N C C P D$, (B) $N C B M C P D_{0.5}$, (C) $N C B M C P D_{1}$, (D) $N C B M C P D_{2}$, and (E) $\mathrm{NCBMCPD}_{3}$. The crystal structure of cellulose type I was also found in all nanocellulose products. Furthermore, the crystallinity and crystal size of all products increased when compared with those of raw material (Table 2). These results showed the same trend as those nanocelluloses extracted from cellulose paper.

From these $X R D$ results, it can be summarized that nanocellulose from cellulose paper as well as cellulose powder showed the identical crystalline peak as the original celluloses but with higher intensity. This can be clarified that the ball-milling pretreatment followed by the mild acid 
hydrolysis can preserve the cellulose allomorph, while the crystallinity and crystal size can be improved from the effective removal of the amorphous region. This could result in higher strength and modulus of elasticity in the obtained nanocellulose [22, 24].

\subsection{Thermogravimetric analysis}

To investigate the thermal stability of samples, thermogravimetric analysis (TGA) was used. The obtained $T G$ and $D T G$ curves of $C P P, B M C P P_{3}, N C C P P, N C B M C P P_{0.5}, N C B M C P P_{1}$, $\mathrm{NCBMCPP}_{2}$, and $\mathrm{NCBMCPP}_{3}$ are shown in Figs. 5 and $\mathbf{6}$, respectively. The thermal degradation data and the residual weight at $600{ }^{\circ} \mathrm{C}$ are tabulated in Table 3. For all samples, the initial weight loss started from room temperature $\left(30{ }^{\circ} \mathrm{C}\right)$ to $125{ }^{\circ} \mathrm{C}$ with a mass loss about $5 \mathrm{wt} \%$. This small weight loss corresponded to the evaporation of adsorbed water and/or the low molecular weight compounds on the surface [25]. For cellulose paper $(C P P)$, the main thermal decomposition occurred at around $270-410{ }^{\circ} \mathrm{C}$ with the maximum degradation temperature of $370{ }^{\circ} \mathrm{C}$ and $87 w t \%$ of weight loss. This stage related to the degradation of cellulosic material.

The remaining residue at $600{ }^{\circ} \mathrm{C}$ was about $6 \mathrm{wt} \%$. For $B M C P P_{3}$, the characteristic of thermal degradation peak was similar to that of $C P P$, which consisted of one decomposition stage at $360{ }^{\circ} \mathrm{C}$. This can be concluded that the ball milling had no significant effect on the thermal stability of cellulose paper. In contrast, for nanocellulose products, $N C C P P, N C B M C P P_{0.5}$, 
$N C B M C P P_{1}, N C B M C P P_{2}$, and $N C B M C P P_{3}$, the decrease in the temperature of the maximum mass loss rate was found at around $200-420{ }^{\circ} \mathrm{C}$ with the maximum degradation temperature around $340-350{ }^{\circ} \mathrm{C}$ and around $75 \mathrm{wt} \%$ of weight loss. This is because the cellulose paper and ball-milled cellulose before acid hydrolysis contains more amorphous region when compared to its nanocellulose products, as supported by $X R D$ results. In other way, the decreasing of particle size to nanometer led to the increasing of surface area which made thermal degradation easily occurred at lower temperature [26]. Moreover, the remaining residues at $600{ }^{\circ} \mathrm{C}$ for nanocellulose products were about 11-15wt\% which are higher than those of cellulose paper and its ball-milled product. This implied that most of the crystalline region of nanocellulose product performed as a flame resistant at high temperature. It is similar as the results reported by Mandal and Chakrabarty, in which the $T G$ of nanocellulose from sugarcane bagasse showed the main thermal degradation at $373{ }^{\circ} \mathrm{C}$, while that of cellulose started degradation at $385{ }^{\circ} \mathrm{C}$ [19]. Moreover, the remaining residues were $15.58 w t \%$ for nanocellulose and $7.68 w t \%$ for cellulose. $T G$ and $D T G$ curves of $C P D, B M C P D_{3}, \quad N C C P D, N C B M C P D_{0.5}, \quad N C B M C P D_{1}$, $N C B M C P D_{2}$, and $N C B M C P D_{3}$ are also shown in Fig. $S-5$ and Fig. S-6, respectively. The thermal properties are also tabulated in Table 3. The characteristic curve of $C P D$ had no significantly different from that of $C P P$ because of their similar cellulose-based structure. The main thermal degradation occurred at $200-410{ }^{\circ} \mathrm{C}$ with the maximum decomposition temperature 
of $372{ }^{\circ} \mathrm{C}$ and a mass loss of $80 \mathrm{wt} \%$. Meanwhile, $B M C P D_{3}$ showed the parallel curves with a little lower value of $360{ }^{\circ} \mathrm{C}$ in the maximum thermal degradation peak and the mass loss of 80 $w t \%$. From this result, it can be also concluded that the pretreating of cellulose powder by planetary ball-milling with $300 \mathrm{rpm}$ in $3 \mathrm{~h}$ had no significant effect on the thermal stability of cellulose powder. For the nanocellulose, the maximum decomposition temperature was decreased to $300-330{ }^{\circ} \mathrm{C}$ with the mass loss around $75 \mathrm{wt} \%$. The remaining of residue at $600{ }^{\circ} \mathrm{C}$ was around $14 w t \%$ which was higher than that of raw cellulose powder due to the higher crystallinity of nanocellulose.

From $T G$ and $D T G$ results, it can be concluded that nanocellulose from hydrolysis of cellulose or ball-milled cellulose by using $47 w t \%$ sulfuric acid in mild condition had higher thermal stability at high temperature when comparing with the raw cellulose material. This phenomenon should be resulted from the higher of crystallinity of nanocellulose which acted as the flame resistant at high temperature [19].

\section{4.\#onclusions}

Ball milling pretreatment followed by mild acid hydrolysis can extract nanocellulose from cellulose paper and cellulose powder with a high yield. The pretreated cellulose with ball milling showed the smaller diameter size which can promote the mild acid hydrolysis. The morphologies 
of nanocelluloses were significantly different from cellulose materials by decreasing to nanometer scale without any new chemical composition occurred. Comparing with the cellulose and ball-milled cellulose materials, the crystallinity and crystal size of nanocellulose increased due to the removal of amorphous phase from the cellulose structure. The maximum decomposition temperature of nanocellulose became lower but the remaining residue over $600{ }^{\circ} \mathrm{C}$ was higher than those of cellulose and ball-milled cellulose materials. The present method was identified to be useful for the extraction of nanocellulose from cellulose with high yield and quality.

\section{Acknowledgements}

This work is supported by JST and Aomori City Government. Phanthong P. gratefully acknowledges the scholarship from the Ministry of Education, Culture, Sports, Science and Technology (MEXT) of Japan.

\section{References}

[1] Siqueira G, Bras J, Dufresne A. Cellulosic bionanocomposites: a review of preparation, properties and applications. Polymers 2010;2:728-765. 
[2] Peng BL, Dhar N, Liu HL, Tam KC. Chemistry and applications of nanocrystalline cellulose and its derivatives: a nanotechnology perspective. Can J Chem Eng 2011;89:1191-1206.

[3] Moon RJ, Martini A, Nairn J, Simonsen J, Youngblood J. Cellulose nanomaterials review: structure, properties and nanocomposites. Chem Soc Rev 2011;40:3941-3994.

[4] Habibi Y, Lucia LA, Rojas OJ. Cellulose nanocrystals: chemistry, self-Assembly, and applications. Chem Rev 2010;110:3479-3500.

[5] Lu A, Fan L, Zheng H, Lu Q, Liao Y, Huang B. Preparation, characterization and optimization of nanocellulose whiskers by simultaneously ultrasonic wave and microwave assisted. Bioresource Technol 2013;146:82-88.

[6] Dong XM, Revol JF, Gray DG. Effect of microcrystallite preparation conditions on the formation of colloid crystals of cellulose. Cellulose 1998;5:19-32.

[7] Haafiz MKM, Hassan A, Zakaria Z, Inuwa IM. Isolation and characterization of cellulose nanowhiskers from oil palm biomass microcrystalline cellulose. Carbohyd Polym 2014;103:119-125.

[8] Barakat A, Mayer-Laigle C, Solhy A, Arancon RAD, Vries HD, Luque R. Mechanical pretreatments of lignocellulosic biomass: towards facile and environmentally sound technologies for biofuels production. RSC Adv 2014;4:48109-48127. 
[9] Kim HJ, Lee S, Kim J, Mitchell RJ, Lee JH. Environmentally friendly pretreatment of plant biomass by planetary and attrition milling. Bioresource Technol 2013;144:50-56.

[10] Baheti VK, Abbasi R, Militky J. Ball milling of jute fibre wastes to prepare nanocellulose. World J Eng 2012;9:45-50.

[11] Avolio R, Bonadies I, Capitani D, Errico ME, Gentile G, Avella M. A multitechnique approach to assess the effect of ball milling on cellulose. Carbohyd Polym 2012;87:265-273.

[12] Feng YT, Han K, Owen DRJ. Discrete element simulation of the dynamics of high energy planetary ball milling processes. Mater Sci Eng A 2004;375-377:815-819.

[13] Ouajai S, Shanks RA. Solvent and enzyme induced recrystallization of mechanically degraded hemp cellulose. Cellulose 2006;13:31-44.

[14] Terinte N, Ibbett R, Schuster KC. Overview on native cellulose and microcrystalline cellulose I structure studied by x-ray diffraction (WAXD): comparison between measurement techniques. Lenzinger Ber 2011;89:118-131.

[15] Revol JF, Dietrich A, Goring DAI. Effect of mercerization on the crystallite size and crystallinity index in cellulose from different sources. Can J Chem 1987;65:1724-1725.

[16] Rhim JW. Effect of PLA lamination on performance characteristics of agar/א-carrageenan/clay bio-nanocomposite film. Food Res Int 2013;51:714-722. 
[17] Bondeson D, Mathew A, Oksman K. Optimization of the isolation of nanocrystals from microcrystalline cellulose by acid hydrolysis. Cellulose 2006;13:171-180.

[18] Agbor VB, Cicek N, Sparling R, Berlin A, Levin DB. Biomass pretreatment: fundamentals toward application. Biotechnol Adv 2011;29:675-685.

[19] Mandal A, Chakrabarty D. Isolation of nanocellulose from waste sugarcane bagasse (SCB) and its characterization. Carbohyd Polym 2011;86:1291-1299.

[20] Li R, Fei J, Cai Y, Li Y, Feng J, Yao J. Cellulose whiskers extracted from mulberry: a novel biomass production. Carbohyd Polym 2009;76:94-99.

[21] Lu P, Hsieh YL. Preparation and properties of cellulose nanocrystals: rods, spheres, and network. Carbohyd Polym 2010;82:329-336.

[22] Peng Y, Gardner DJ, Han Y, Kiziltas A, Cai Z, Tshabalala MA. Influence of drying method on the material properties of nanocellulose I: thermostability and crystallinity. Cellulose $2013 ; 20: 2379-2392$.

[23] Johar N, Ahmad I, Dufresne A. Extraction, preparation and characterization of cellulose fibres and nanocrystals from rice husk. Ind Crop Prod 2012;37:93-99.

[24] Lavoine N, Desloges I, Dufresne A, Bras J. Microfibrillated cellulose - Its barrier properties and applications in cellulosic materials: A review. Carbohyd Polym 2012;90:735-764. 
[25] Santos RMD, Neto WPF, Silverio HA, Martins DF, Dantas NO, Pasquini D. Cellulose nanocrystals from pineapple leaf, a new approach for the reuse of this agro-waste. Ind Crop Prod 2013;50:707-714.

[26] Abraham E, Deepa B, Pothen LA, Cintil J, Thomas S, John MJ, Anandjiwala R, Narine SS. Environmental friendly method for the extraction of coir fibre and isolation of nanofiber.

Carbohyd Polym 2013;92:1477-1483.

\section{Table captions}

Table 1 Nanocellulose product yields

Table 2 Crystallinity and crystal size of samples

Table 3 Thermal properties of samples

\section{Figure captions}

Fig. $1 S E M$ images of (A) $C P P$, (B) $B M C P P_{0.5}$, (C) $B M C P P_{1}$, (D) $B M C P P_{2}$, (E) $B M C P P_{3}$, (F) $N C C P P,(\mathrm{G}) N C B M C P P_{0.5},(\mathrm{H}) N C B M C P P_{1},(\mathrm{I}) N C B M C P P_{2}$, and (J) $N C B M C P P_{3}$.

Fig. 2 FTIR spectra of (A) $C P P$, (B) $B M C P P_{3},(\mathrm{C}) N C C P P$, (D) $N C B M C P P_{0.5}$, (E) $N C B M C P P_{1}$, (F) $N C B M C P P_{2}$, and (G) $N C B M C P P_{3}$. 
Fig. $3 X R D$ patterns of (A) $C P P$, (B) $B M C P P_{0.5}$, (C) $B M C P P_{1}$, (D) $B M C P P_{2}$, and (E) $B M C P P_{3}$. Fig. $4 X R D$ patterns of (A) $N C C P P$, (B) $N C B M C P P_{0.5}$, (C) $N C B M C P P_{1}$, (D) $N C B M C P P_{2}$, and (E) $\mathrm{NCBMCPP}$.

Fig. $5 T G$ curves of $C P P, B M C P P_{3}, N C C P P, N C B M C P P_{0.5}, N C B M C P P_{1}, N C B M C P P_{2}$, and $N C B M C P P_{3}$.

Fig. $6 D T G$ curves of $C P P, B M C P P_{3}, N C C P P, N C B M C P P_{0.5}, N C B M C P P_{1}, N C B M C P P_{2}$, and $\mathrm{NCBMCPP}{ }_{3}$ 
Figure 1

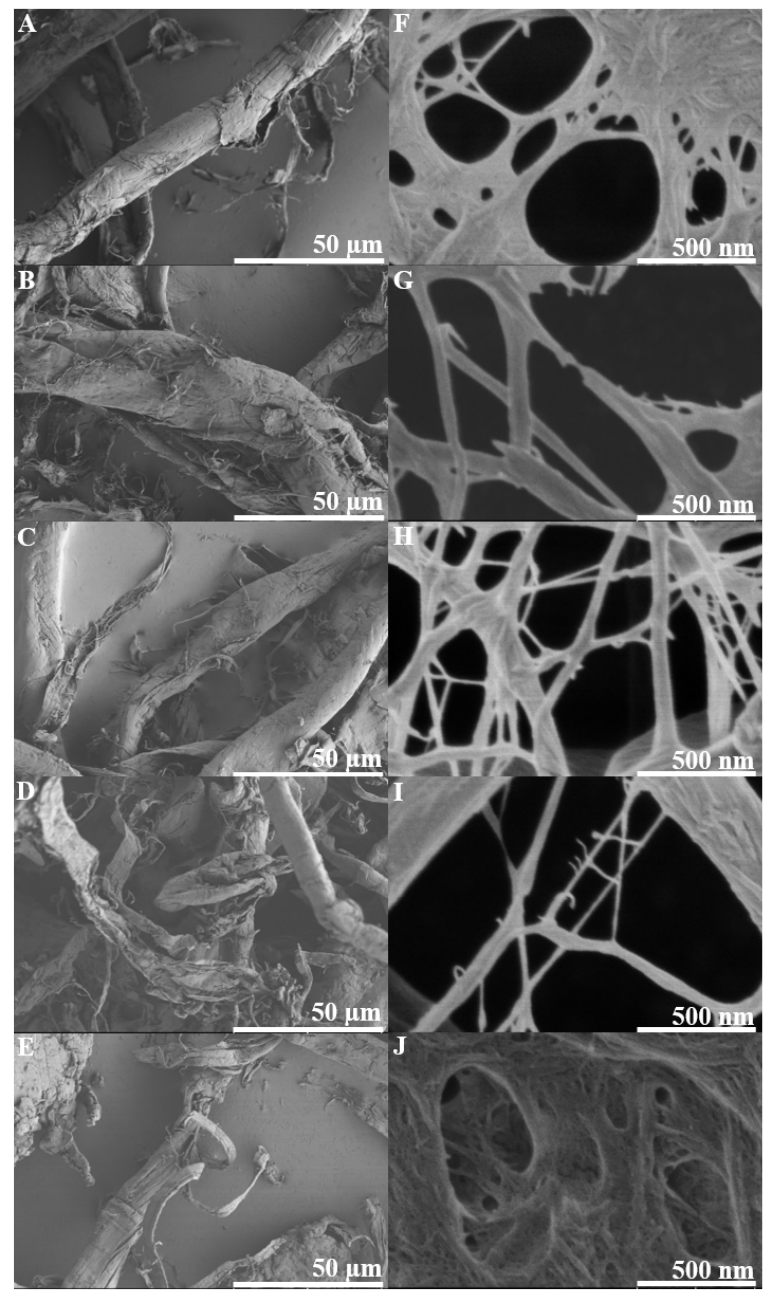


Figure 2

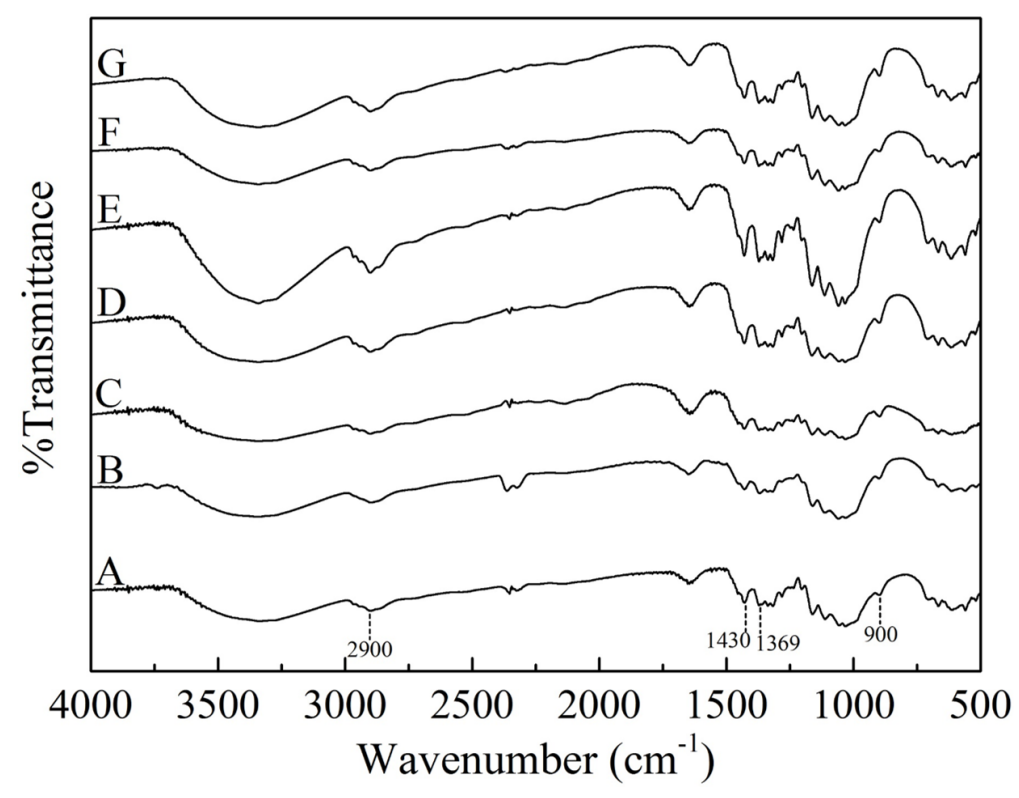


Figure 3

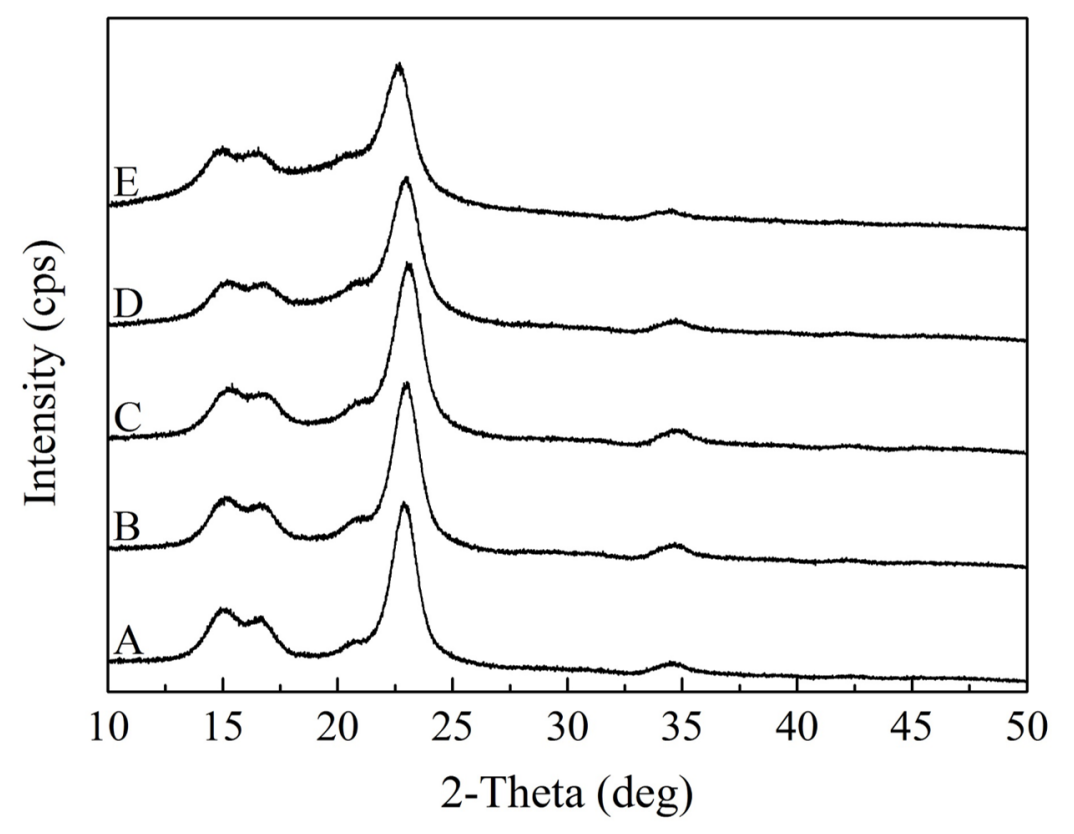


Figure 4

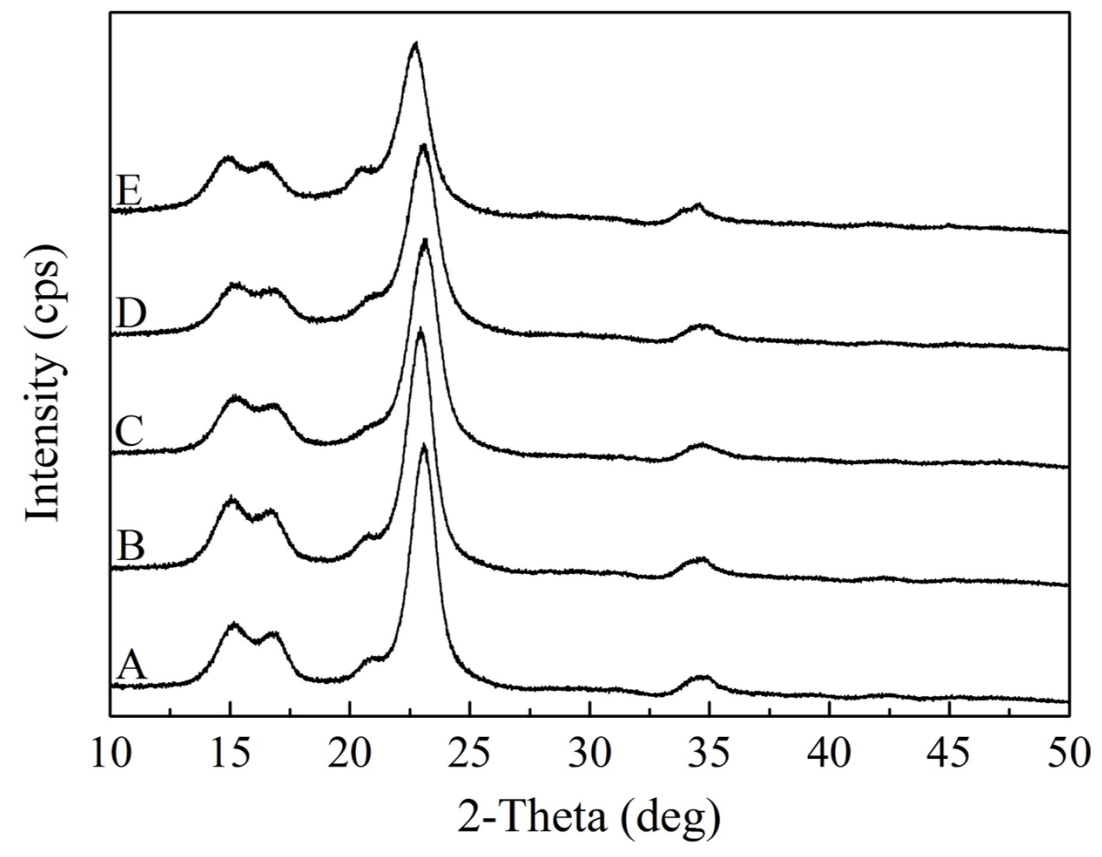


Figure 5

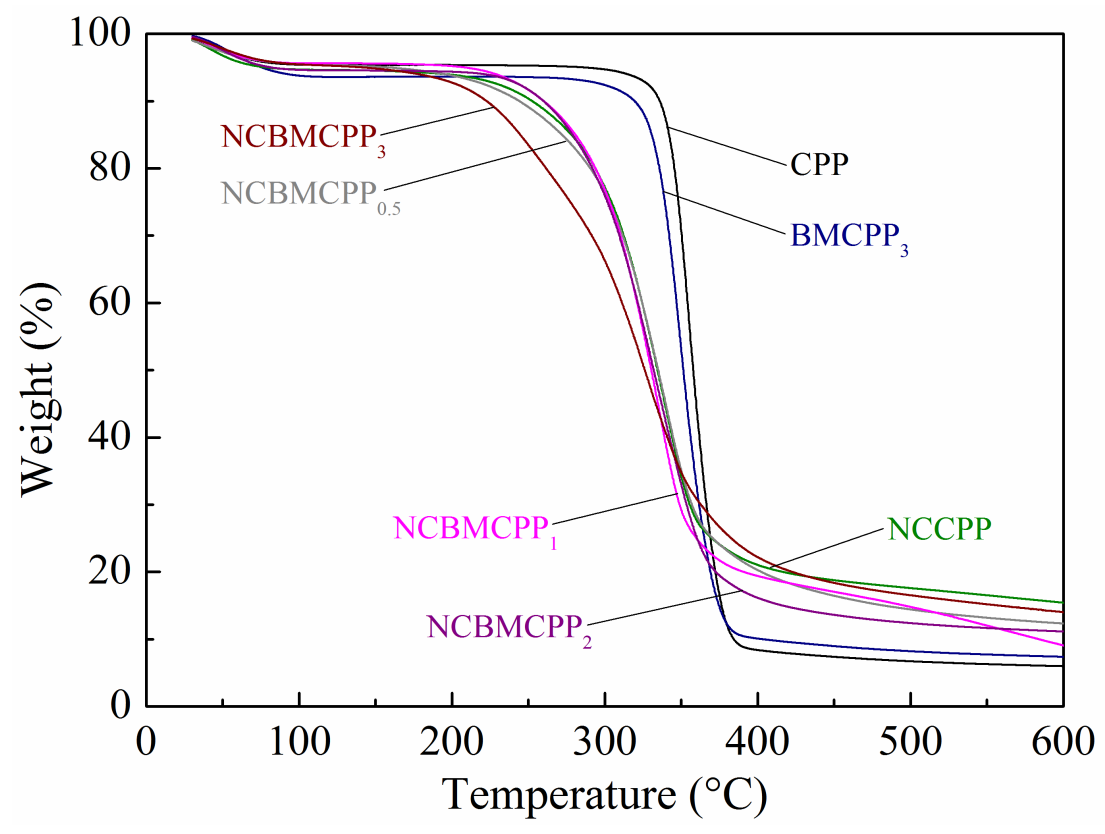


Figure 6

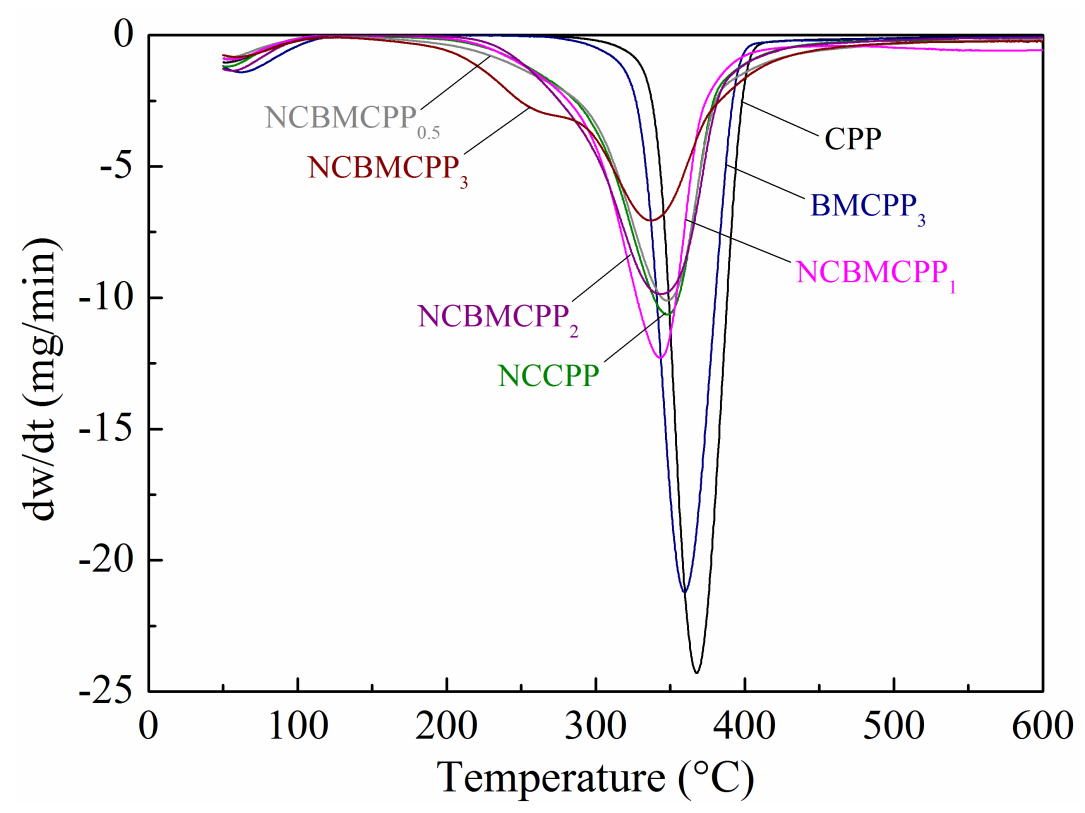


Table 1

\begin{tabular}{lc}
\hline Products & Yield (\%) \\
\hline$N C C P P$ & 89.3 \\
NCBMCPP $_{0.5}$ & 84.8 \\
$N_{\text {NCBMCPP }}$ & 87.9 \\
$N_{\text {NCBMCPP }}$ & 86.0 \\
$N_{2}$ & 77.5 \\
\hline$N C C P D$ & 81.1 \\
$N C B M C P D_{0.5}$ & 83.5 \\
$N C B M C P D_{1}$ & 83.1 \\
$N C B M C P D_{2}$ & 83.7 \\
$N C B M C P D_{3}$ & 87.1 \\
\hline
\end{tabular}


Table 2

\begin{tabular}{lcccc}
\hline \multirow{2}{*}{ Materials } & \multicolumn{2}{c}{ Crystallinity (\%) } & \multicolumn{2}{c}{ Crystal size $(\mathrm{nm})$} \\
& Raw materials & $\begin{array}{c}N C \\
\text { Products }\end{array}$ & Raw materials & $\begin{array}{c}\text { NC } \\
\text { Products }\end{array}$ \\
\hline$C P P$ & 91.1 & 94.4 & 12.3 & 12.9 \\
$\mathrm{BMCPP}_{0.5}$ & 89.0 & 93.3 & 11.2 & 12.1 \\
$\mathrm{BMCPP}_{1}$ & 87.4 & 92.7 & 10.5 & 10.8 \\
$B M C P P_{2}$ & 81.0 & 89.5 & 9.5 & 10.4 \\
$B M C P P_{3}$ & 68.6 & 84.8 & 8.5 & 11.1 \\
\hline$C P D$ & 81.8 & 82.7 & 8.1 & 8.1 \\
$B M C P D_{0.5}$ & 67.9 & 79.2 & 5.6 & 7.6 \\
$B M C P D_{1}$ & 56.1 & 69.5 & 3.8 & 5.3 \\
$B M C P D_{2}$ & 33.6 & 65.5 & 2.7 & 4.7 \\
$B M C P D_{3}$ & 18.0 & 59.8 & 1.6 & 3.8 \\
\hline
\end{tabular}


Table 3

\begin{tabular}{|c|c|c|c|c|}
\hline Materials & $\begin{array}{l}\text { Main thermal } \\
\text { degradation } \\
\text { range }\left({ }^{\circ} \mathrm{C}\right)\end{array}$ & $\begin{array}{c}\text { Max } \\
\text { decomposition } \\
\text { temperature in each } \\
\text { stage }\left({ }^{\circ} \mathrm{C}\right) \\
\end{array}$ & $\begin{array}{c}\text { Weight loss in } \\
\text { each stage } \\
(\%)\end{array}$ & $\begin{array}{l}\text { Residual weight (\%) } \\
\text { at } 600{ }^{\circ} \mathrm{C}\end{array}$ \\
\hline$C P P$ & $270-410$ & 370 & 87 & 6 \\
\hline$B M C P P_{3}$ & $270-410$ & 360 & 83 & 7 \\
\hline NCCPP & $200-420$ & 350 & 74 & 15 \\
\hline$N_{C B M C P} P_{0.5}$ & $200-420$ & 350 & 76 & 12 \\
\hline$N C B M C P P_{1}$ & $200-400$ & 345 & 76 & 9 \\
\hline $\mathrm{NCBMCPP}_{2}$ & $200-420$ & 345 & 78 & 11 \\
\hline \multirow{2}{*}{$N C B M C P P_{3}$} & $200-290$ & 250 & 22 & \multirow{2}{*}{14} \\
\hline & $290-420$ & 340 & 51 & \\
\hline$C P D$ & $200-410$ & 372 & 80 & 12 \\
\hline$B M C P D_{3}$ & $200-400$ & 360 & 80 & 10 \\
\hline NCCPD & $220-450$ & 315 & 62 & 18 \\
\hline$N C B M C P D_{0.5}$ & $210-470$ & 330 & 74 & 15 \\
\hline$N C B M C P D_{1}$ & $210-470$ & 325 & 78 & 12 \\
\hline \multirow{2}{*}{$\mathrm{NCBMCPD}_{2}$} & $200-290$ & 270 & 26 & \multirow{2}{*}{14} \\
\hline & $290-470$ & 325 & 48 & \\
\hline \multirow{2}{*}{$\mathrm{NCBMCPD}_{3}$} & $210-340$ & 300 & 50 & \multirow{2}{*}{12} \\
\hline & $340-470$ & 365 & 25 & \\
\hline
\end{tabular}




\section{Graphical abstract}

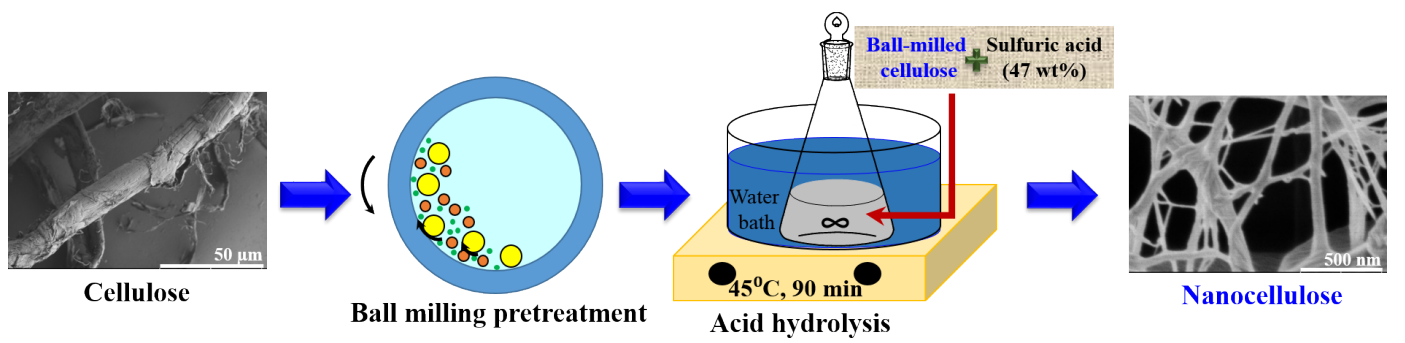

